\title{
Spatially resolved scanning tunneling spectroscopy on single-walled carbon nanotubes
}

\author{
L. C. Venema, J. W. Janssen, M. R. Buitelaar, * J. W. G. Wildöer, S. G. Lemay, L. P. Kouwenhoven, and C. Dekker \\ Department of Applied Sciences and DIMES, Delft University of Technology, Lorentzweg 1, 2628 CJ Delft, The Netherlands
}

(Received 24 January 2000)

\begin{abstract}
Scanning tunneling microscope spectroscopy is used to study in detail the electronic band structure of carbon nanotubes as well as to locally investigate electronic features of interesting topological sites such as nanotube ends and bends. From a large number of measurements of the tunneling density-of-states (DOS) nanotubes can be classified, according to predictions, as either semiconducting (two-third of the total number of tubes) or metallic (one-third). The energy subband separations in the tunneling DOS compare reasonably well to theoretical calculations. At nanotube ends, spatially resolved spectra show additional sharp conductance peaks that shift in energy as a function of position. Spectroscopy measurements on a nanotube kink suggest that the kink is a heterojunction between a semiconducting and a metallic nanotube.
\end{abstract}

\section{INTRODUCTION}

The electronic and atomic structure of a carbon nanotube are related to each other in a remarkable way. A nanotube can be thought of as a rolled up graphene sheet where two parameters, the chirality $\phi$ of the hexagonal lattice along the nanotube shell and the diameter $d$, fully describe its atomic structure. ${ }^{1}$ The indices $(n, m)$ of a nanotube correspond to a specific combination of chiral angle $\phi$ and diameter $d$. Depending critically on these $(n, m)$ indices, a nanotube can be either semiconducting or metallic. ${ }^{2-4}$ Of all possible $(n, m)$ nanotubes, roughly one-third is predicted to be metallic.

Scanning tunneling microscopy (STM) has proven to be a very useful technique to investigate carbon nanotubes since both the atomic and electronic structure can be investigated for individual nanotubes. ${ }^{5-14}$ Atomically resolved imaging enabled us to observe the chiral structure of a nanotube. In addition, the electronic density-of-states (DOS) can be measured for the same tube by scanning tunneling spectroscopy (STS). The DOS of carbon nanotubes in theory consists of a number of one-dimensional (1D) subbands due to the quantization of available energy modes in the circumferential direction. $^{2-4}$ This may be seen, for example, in the theoretically calculated DOS for a semiconducting and a metallic nanotube in Fig. 2 (curves 1 and 6, respectively). The subband onsets are sharp Van Hove singularities due to the onedimensional nature of the subbands. The energy intervals at which the subband onsets appear depend on whether the tube is metallic or semiconducting and on the nanotube diameter. ${ }^{15,16}$ Recent STS measurements verified the general features predicted for the DOS. Nanotubes were found to be either semiconducting or metallic and also Van Hove singularities could be observed. ${ }^{10,11,14}$ Attention is now directed towards the electronic structure of topologically interesting sites in nanotubes, such as ends, bends, and kinks. These sites can be studied spatially resolved by STS. Carrol et al. ${ }^{9}$ observed sharp STS resonances at the tip of multiwalled nanotubes that could be associated with a particular cap structure. Recently, Kim et al. ${ }^{14}$ also observed peaks in the DOS at the end of a metallic single-walled nanotube. They attributed this result to a specific arrangement of pentagons at the cap. Another interesting topological effect is a junction between two nanotubes with different $(n, m)$ numbers, which appears as a sharp bend, or "kink." Section III B discusses a measurement on a sharply bent nanotube that appears from spectroscopy curves above and below the kink to be a molecular semiconductor-metal heterojunction.

In this paper we review results from a large number of STS measurements on individual nanotubes. The main features of the electronic DOS as well as locally resolved data on tube ends and a tube kink are discussed in Sec. III. The following section first gives details of the sample and measurement methods.

\section{EXPERIMENTAL DETAILS}

Measurements were performed on samples of $\mathrm{Au}$ (111) surfaces with single-walled carbon nanotubes that were synthesized by laser evaporation (by Smalley and co-workers). ${ }^{17}$ The Au (111) was prepared by melting pieces of about $30 \mathrm{~mm}^{3}$ from a $99.99 \%$ pure gold wire in a gas flame. The single-crystalline (111) facets appearing on the surface during cooling were used as substrates. Pieces of nanotube soot were dispersed in dichloroethane and ultrasonically agitated to unravel the bundles of tubes. A droplet of the dispersion was then deposited on the substrates of $\mathrm{Au}$ (111) in ambient conditions. The measurements were done in a homebuilt $4 \mathrm{~K}$ STM. STM tips were mechanically cut from a $\operatorname{Pt}(90 \%)$ $\operatorname{Ir}(10 \%)$ wire. Imaging was done in constant-current mode at typical parameters of $60 \mathrm{pA}$ for the tunnel current $I$ and 0.1 $\mathrm{V}$ for the bias voltage $V$.

Some examples of nanotubes imaged with STM at room temperature Fig. 1(a) and at 4 K Figs. 1(b)-1(f) are shown. The scale bar in Figs. 1(b)-1(d) corresponds to $10 \mathrm{~nm}$. The apparent width of the nanotubes varies between 5 and $20 \mathrm{~nm}$. This scale is determined by the size of the STM tip rather than the nanotube diameter, which is in the range of $1.4 \mathrm{~nm}$. Various nanotubes and small bundles can be seen in the image of Fig. 1(a). Carbon nanotubes usually lie straight on the surface but may be deformed due to irregularities in the surface such as monoatomic steps or crossings with other tubes. Hertel et al. showed that single-walled carbon nanotubes may experience substantial deformations at surface irregularities due to the large van der Waals binding energies be- 
a

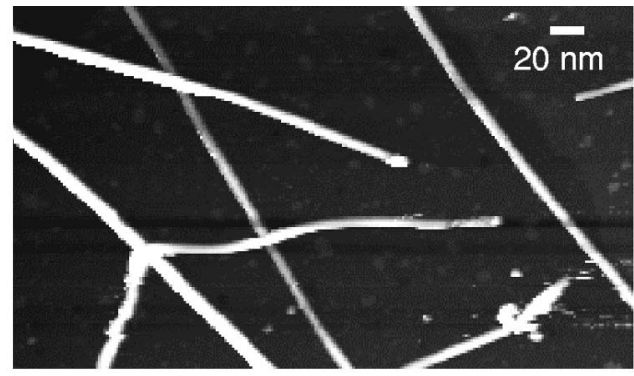

b

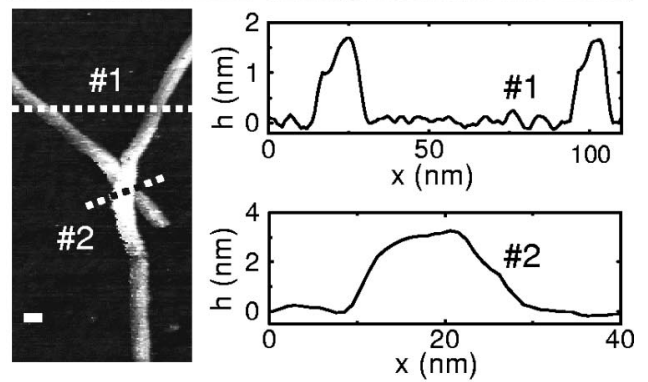

C

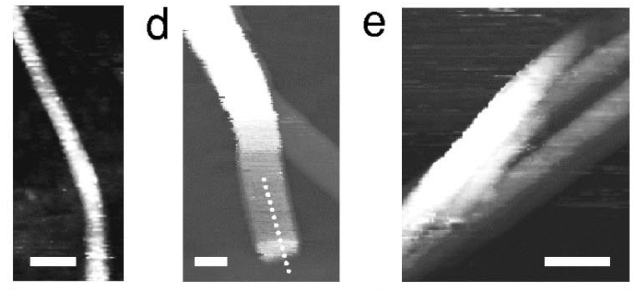

f

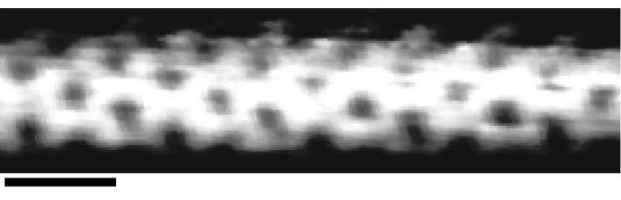

$0.5 \mathrm{~nm}$

FIG. 1. STM images at room temperature (a), and at $4 \mathrm{~K}$ (b)-(f). The scale bar in b-e is $10 \mathrm{~nm}$. (a) Large-scale image where various nanotubes and small bundles on a surface of $\mathrm{Au}(111)$ can be observed. (b) Crossing of two nanotubes. The curves on the right are two height profiles taken along the dashed lines indicated in the image. $h$ is the height, $x$ is the coordinate along the lines. (c) A sharply bent nanotube. (d) An end of a nanotube that splits off from a bundle. The dotted line illustrates how a series of STS curves can be taken to observe gradual changes in the local DOS at the end. (e) A bundle in which individual nanotubes can be discerned. (f) Atomically resolved image of a chiral nanotube. The image is corrected for the geometrical distortion that stretches the apparent nanotube lattice in the direction normal to the tube axis (Ref. 21.

tween nanotubes and the substrate surface. ${ }^{18}$ The nanotube emerging from the left-bottom corner in Fig. 1(a) is indeed bent strongly at the crossings with two other nanotubes. The total lateral extent of such bends is typically about $30 \mathrm{~nm}$. The STM images of Figs. 1(b)-1(e) show details of, respectively, a crossing, a sharp bend, a nanotube end and a bundle in which several individual nanotubes can be resolved. The right part of Fig. 1(b) are two height profiles $h(x)$ that are taken along the dashed lines shown in the STM image on the left. From the top profile, the individual nanotubes have an apparent height relative to the surface of 1.70 and $1.65 \mathrm{~nm}$. Taking into account a van der Waals distance between the tubes and the surface of about $0.25 \mathrm{~nm},{ }^{19}$ the diameters are 1.45 and $1.40 \mathrm{~nm}$. The crossing of the two tubes, shown in the bottom $h(x)$ profile, has a total height of $3.35 \mathrm{~nm}$. This corresponds to the sum of the individual tube heights and a van der Waals distance between the nanotubes and between the lower nanotube and the surface. Figure 1(c) is an image of a sharply bent nanotube, or possibly of a nanotube "kink." As mentioned in the introduction, kinks can be junctions between different nanotubes. Figure $1(\mathrm{~d})$ is zoomed in on a nanotube that splits off from a small bundle. The dotted line crossing the end of the nanotube is an example of a line along which a series of spectroscopy measurements can be taken to observe the change in the local DOS at the end of the tube. Such measurements are discussed in Sec. III B. Figure 1(e) shows a bundle of nanotubes in which the individual nanotubes can be discerned. Figure $1(\mathrm{f})$ is an atomically resolved image of a chiral nanotube. The image has been corrected for the geometric distortion that stretches the apparent carbon lattice in the direction perpendicular to the tube. ${ }^{20,21}$ The chiral angle obtained from Fig. 1(f) is $21 \pm 1^{\circ}$. In general, a large variety of chiral angles could be found for this nanotube material, ranging from zigzag to armchair. Diameters that are measured from apparent height measurements are in general quite small, about $0.9 \mathrm{~nm}$, which is probably due to the flattening of nanotubes by tip forces during imaging. ${ }^{21}$ More reliable values can be obtained from tunneling spectroscopy measurements. As will be discussed in detail below, the energy differences between the subbands in the DOS depend strongly on the diameter. Using this to deduce the diameter, values of $\sim 1.1-1.5 \mathrm{~nm}$ are found, which corresponds reasonably well to TEM measurements where diameters of about $1.4 \mathrm{~nm}$ were determined. ${ }^{17}$

All spectroscopy measurements presented in this paper were done at $4 \mathrm{~K}$ and on individual nanotubes. Currentvoltage $(I-V)$ tunnel spectra were taken by switching off the feedback and recording the current as a function of the bias voltage while keeping the STM tip at a fixed position. $d I / d V-V$ curves (from here called $d I / d V$ curves) were numerically calculated from $I-V$ spectra. $d I / d V$ can be taken as a measure for the DOS.

\section{EXPERIMENTAL RESULTS}

\section{A. Density-of-States}

\section{Long nanotubes}

Two classes of DOS are expected for nanotubes, one for semiconducting and one for metallic nanotubes. The curves 1 and 6 in Fig. 2 represent the theoretical DOS for a $(11,7)$ and a $(9,9)$ tube, which have approximately the same diameter but are semiconducting and metallic, respectively. Two types of $d I / d V$ spectra taken with STM can indeed be distinguished for various nanotubes. Several examples are shown in Fig. 2. The left figure shows $d I / d V$ curves $2-5$ with various peaks and a small energy gap $\Delta E_{\text {gap }}$ of about $0.7 \mathrm{~V}$. The right figure, curves 7-10, have only two clear peaks or onsets in the displayed voltage range and have a large plateau of constant conductance around zero bias $\Delta E_{s u b}$ of about 1.9 $\mathrm{V}$ in width. The experimentally obtained spectra compare well to the theoretical curves. Both energy differences $\Delta E_{g a p}$ and $\Delta E_{\text {sub }}$ should scale in theory inversely linear with the diameter of a nanotube. Semiconducting nanotubes with a diameter of $1.4 \mathrm{~nm}$ have an energy gap $\Delta E_{g a p}=2 d_{n n} \gamma_{0} / d$ 

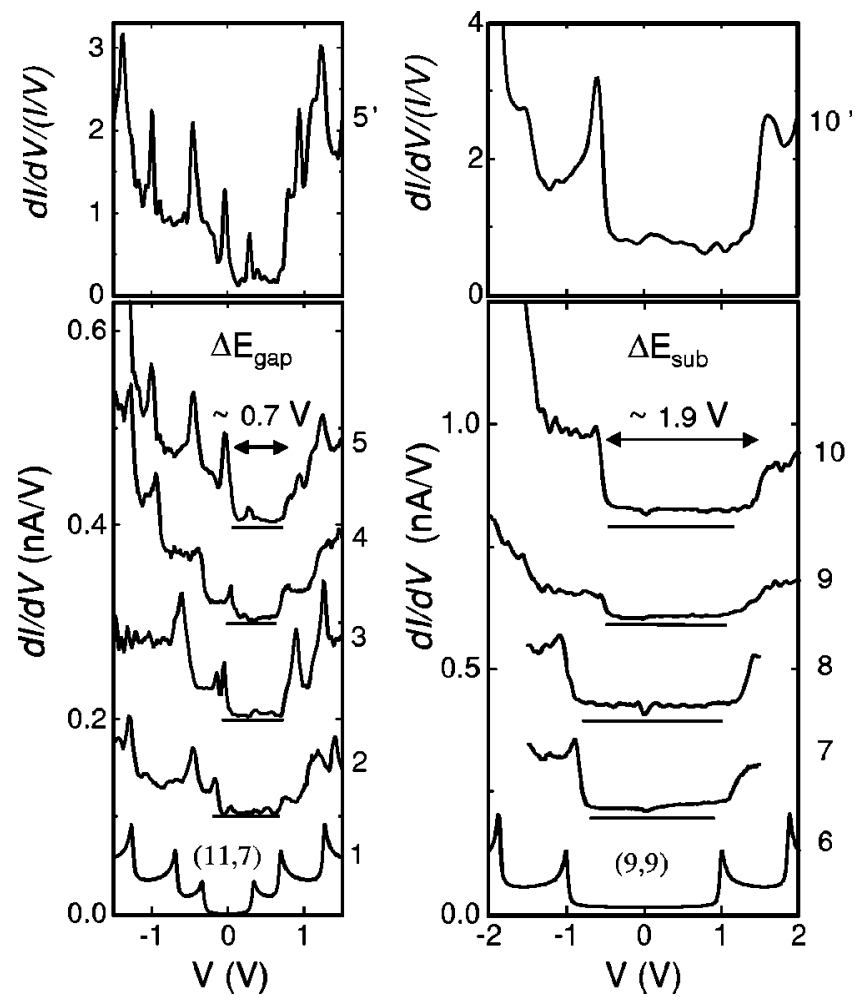

FIG. 2. Experimental and theoretical DOS for semiconducting (left) and metallic (right) nanotubes. For clarity the curves are vertically offset by 0.1 and $0.2 \mathrm{nA} / \mathrm{V}$, respectively. The DOS consists of a number of subbands with sharp (Van Hove) singularities at the onsets. Curves 1 and 6 represent the theoretical DOS for a $(11,7)$ and a $(9,9)$ nanotube, which are semiconducting and metallic, respectively. These curves were calculated by Philippe Lambin. Curves 2-5 are $d I / d V$ spectra taken by STM on nanotubes that can be identified as semiconducting and have an energy gap $\Delta E_{\text {gap }}$ of about $0.7 \mathrm{~V}$. Curves $7-10$ are spectra on nanotubes that can be identified as metallic and have a large plateau of constant conductance $\Delta E_{s u b}$ of about $1.9 \mathrm{~V}$. The curves 5' and 10' in the top parts are normalized curves, obtained from 5 and 10 .

$\approx 0.6 \mathrm{eV}$, where $d_{n n}=1.42 \AA$ is the distance between nearest-neighbor carbon atoms and $\gamma_{0}=2.9 \mathrm{eV}$ is the energy overlap integral between nearest neighbors. ${ }^{22}$ For metallic nanotubes, the separation $\Delta E_{\text {sub }}$ between the onsets is $\Delta E_{\text {sub }}=6 a \gamma_{0} / d \approx 1.8 \mathrm{eV}$ for $d=1.4 \mathrm{~nm}$. In this case, there is no energy gap but the DOS between the subbands is finite (albeit small) and constant. The experimentally obtained $\Delta E_{\text {gap }}$ and $\Delta E_{\text {sub }}$ correspond to these calculated values. Out of 29 nanotubes on which $d I / d V$ spectra have been obtained, 19 were semiconducting and 10 were metallic. This result confirms the prediction that about one-third of the $(n, m)$ nanotubes are metallic and two-thirds are semiconducting.

It is well known from STS literature that at large bias voltage the tunnel barrier is reduced considerably, which leads to an overestimated DOS at higher bias. ${ }^{23}$ To account for this effect, $d I / d V$ can be normalized by $I / V$. The top curves $5^{\prime}$ and $10^{\prime}$ in Fig. 2 are normalized spectra, $d I / d V /(I / V)$, calculated from curves 5 and 10 . The normalization technique is especially useful for metallic spectroscopy curves since the first onsets appear at a relatively high voltage, around $\pm 1 \mathrm{~V}$. The peak shapes of the subband onsets are enhanced by the normalization as can be observed

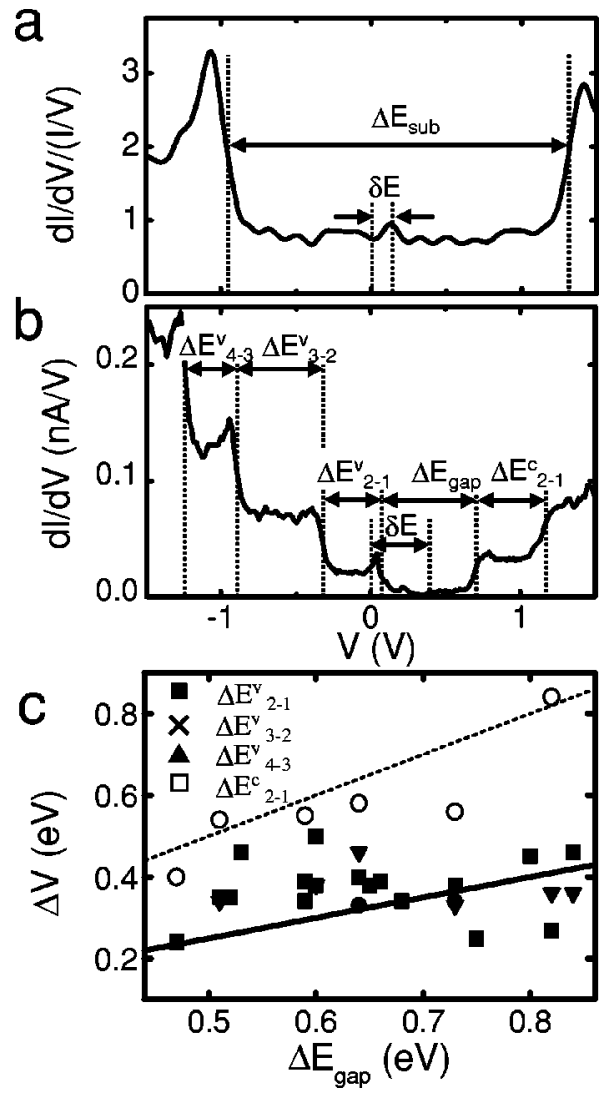

FIG. 3. Analysis of energy intervals between subbands and the shifts of the Fermi level. (a) Metallic $d I / d V /(I / V)$ spectrum in which $\Delta E_{\text {sub }}$ and Fermi level shift $\delta E$ are indicated. (b) Semiconducting $d I / d V$ spectrum with several subband onset separations indicated, $\Delta E_{i-j}^{v}$ for the valence band and $\Delta E_{i-j}^{c}$ for the conductance band. The shift of the Fermi level $\delta E$ is also shown. (c) Subband intervals $\Delta E_{i-j}^{v, c}$ in voltage differences $\Delta V$ plotted as a function of the energy gaps for about 20 semiconducting nanotubes. The solid line represents $1 / 2 \Delta E_{\text {gap }}$ and the dotted line $\Delta E_{\text {gap }}$.

for the metallic curve $10^{\prime}$. The value for $\Delta E_{s u b}$ determined from the normalized curves is slightly smaller, by about 0.03 $\mathrm{eV}$, as compared to the value found from $d I / d V$ curves, due to a small shift of the onsets. For the semiconducting curves, the normalization does not significantly affect the intervals between the subband onsets since they are smaller. The normalized curves are smoothed near zero bias because of the large divergences from the normalization procedure. Determining the peak position of the first valence subband onset for the semiconducting curves often is problematic because small noise fluctuations may lead to spurious peaks. We therefore use the normalization technique for metallic nanotubes to obtain the most reliable value for $\Delta E_{s u b}$, but determine the intervals between subband onsets for semiconducting nanotubes from $d I / d V$ curves.

Various energy intervals $\Delta E_{i-j}$, both above and below the Fermi energy, are indicated for the semiconducting curve in Fig. 3(b). The differences between the subband onsets follows within a good estimate a simple rule. ${ }^{15,16}$ From the approximately linear energy dispersion near the Fermi level for graphene, the first four Van Hove singularities for semiconductors can be found to appear at energies $E_{1}=$ $\pm d_{n n} \gamma_{0} / d, E_{2}= \pm 2 d_{n n} \gamma_{0} / d, E_{3}= \pm 4 d_{n n} \gamma_{0} / d$, and $E_{4}=$ $\pm 5 d_{n n} \gamma_{0} / d$. The separations between the singularities are 
then $\quad \Delta E_{2-1}=d_{n n} \gamma_{0} / d=1 / 2 \Delta E_{\text {gap }}, \quad \Delta E_{3-2}=2 d_{n n} \gamma_{0} / d$ $=\Delta E_{\text {gap }}$, and $\Delta E_{4-3}=d_{n n} \gamma_{0} / d=1 / 2 \Delta E_{\text {gap }}$. For a metal, the Van Hove singularities appear at energies $E_{1}=$ $\pm 3 d_{n n} \gamma_{0} / d, E_{2}= \pm 6 d_{n n} \gamma_{0} / d$, and $E_{3}= \pm 9 d_{n n} \gamma_{0} / d$. The separations $\Delta E_{i-j}$ are therefore all of the size $3 d_{n n} \gamma_{0} / d$ $=\Delta E_{s u b}$. It is difficult to observe several energy intervals in the metallic case since the onsets appear at large energies $[|V| \gtrsim 1 \mathrm{~V}$, see for example Fig. 3(a)]. However, in the semiconducting spectra, three to five subband onsets are usually visible. In Fig. 3(c), energy differences that were obtained for about 20 semiconducting nanotubes are plotted against the energy gap of each tube and compared with $\Delta E_{\text {gap }}$ (dotted line) and $1 / 2 \Delta E_{\text {gap }}$ (solid line). There is much scatter in the data, but it can be observed that the first and third subband separations (closed squares, circles, and triangles) are near the $1 / 2 \Delta E_{\text {gap }}$ line whereas the second subband separations (open circles) are closer to the $\Delta E_{\text {gap }}$ line. This observation is in agreement with the simple rule of alternating subband spacings. Deviations in the positions of the subband onsets may occur due to the nonlinearity of the graphene energy dispersion far away from the Fermi energy and due to hybridization with the gold substrate.

Also indicated in Figs. 3(a) and 3(b) is the shift $\delta E$ of the spectra relative to zero bias. This shift can be attributed to the charge transfer between the gold-nanotube interface due to the difference in work function. Au has a work function of $\sim 5.3 \mathrm{eV}$, whereas nanotubes were found to have a work function of about $4.3 \mathrm{eV} .^{24,25}$ The aligning of the Fermi levels by transfer of electrons from nanotube to gold leads to an asymmetric position of the nanotube band structure relative to the Fermi level. From the STS spectra we find that $\partial E$ is about $0.3 \pm 0.1 \mathrm{eV}$ for both metallic and semiconducting nanotubes, independent of $\Delta E_{\text {gap }}$ and $\Delta E_{\text {sub }}$. This agrees with the theoretical Fermi-level shift of about $0.2 \mathrm{eV}$ as a result of the charge transfer between a metallic substrate and a nanotube. ${ }^{26}$ In the case of the semiconducting nanotubes the Fermi level is actually shifted into the valence band. For metallic tubes the Fermi level stays well within the plateau between the subband onsets $\Delta E_{\text {sub }}$.

Often, a suppression of the tunneling conductance near zero bias can be observed, see for example the metallic $d I / d V$ curves 7 and 10 in Fig. 2. This zero bias anomaly is also sometimes present in spectra taken on the bare gold substrate away from the nanotubes. The suppression of the tunneling DOS at the Fermi energy for disordered conductors is a well-known problem in the literature. ${ }^{27-29}$ It was argued that electron-electron interactions in the presence of impurities results in a negative correction to the DOS, which is singular at the Fermi energy. This theory may apply to the present case, since the STM tip is likely to be a disordered conductor as it is made from an alloy of Pt and Ir. The zero bias anomaly is possibly enhanced by the Luttinger liquid character of carbon nanotubes. ${ }^{30,31}$

\section{Short nanotubes}

In short nanotubes the DOS may show additional structure due to finite-size effects. For nanotubes of finite length the available energy states in the length direction will be quantized. For finite metallic nanotubes it has been shown

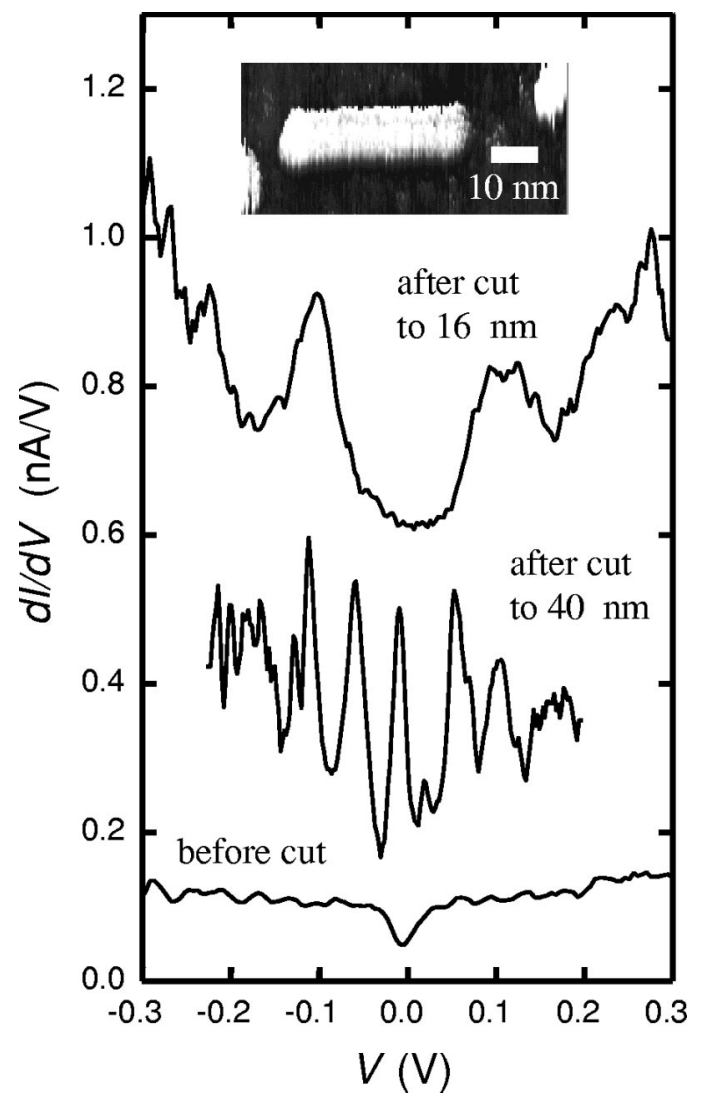

FIG. 4. $d I / d V$ spectra zoomed in at low energies on a metallic nanotube that has been cut into short pieces to observe energy-level splitting. The inset shows a topographic image of the tube after it was cut by voltage pulses to a piece of $40-\mathrm{nm}$. The bottom spectrum was taken on the nanotube before it was cut. The tunneling conductance shows an oscillatory behavior (middle curve) with a period of about $50 \mathrm{mV}$ for the $40-\mathrm{nm}$ piece. After cutting the tube to $16 \mathrm{~nm}$, the period is about $160 \mathrm{mV}$ (top curve). The top curve is offset vertically for clarity by $0.6 \mathrm{nA} / \mathrm{V}$. The middle curve is not offset, but was taken at a smaller tunnel distance and therefore has a higher tunneling conductance.

that the energy bands that cross at the Fermi level consist of well-separated single electron states that are coherent over a considerable length of the nanotube..$^{32-34}$ The energy level splitting $\Delta E_{L}$ scales inversely linear with the length $L$, as $\Delta E_{L}=h v_{F} / 2 L$, where $v_{F}=9 \times 10^{5} \mathrm{~m} / \mathrm{s}$ is the Fermi velocity. Discrete energy levels can be observed by cutting nanotubes into small pieces using voltage pulses to the STM tip. ${ }^{35}$ The inset of Fig. 4 shows a nanotube that was cut into a piece of about $40 \mathrm{~nm}$. Its metallic character is apparent from $d I / d V$ measurements before the cuts, that were similar to the metallic curves in the right plot of Fig. 2. After the nanotube was cut into a piece of $40 \mathrm{~nm}$, the DOS showed oscillatory behavior near the Fermi energy, with a peak-peak separation of about $50 \mathrm{meV}$ (Fig. 4). More pulses were applied to cut the tube to even smaller pieces. $d I / d V$ spectra on a $16-\mathrm{nm}$ piece (Fig. 4 , top curve) also showed clear oscillations, with a peak-peak separation of about $160 \mathrm{meV}$. These values correspond reasonably well with the predicted energy level separation: the theoretical $\Delta E_{L}$ is $50 \mathrm{meV}$ for $L=40 \mathrm{~nm}$ and $120 \mathrm{meV}$ for $L=16 \mathrm{~nm}$. 


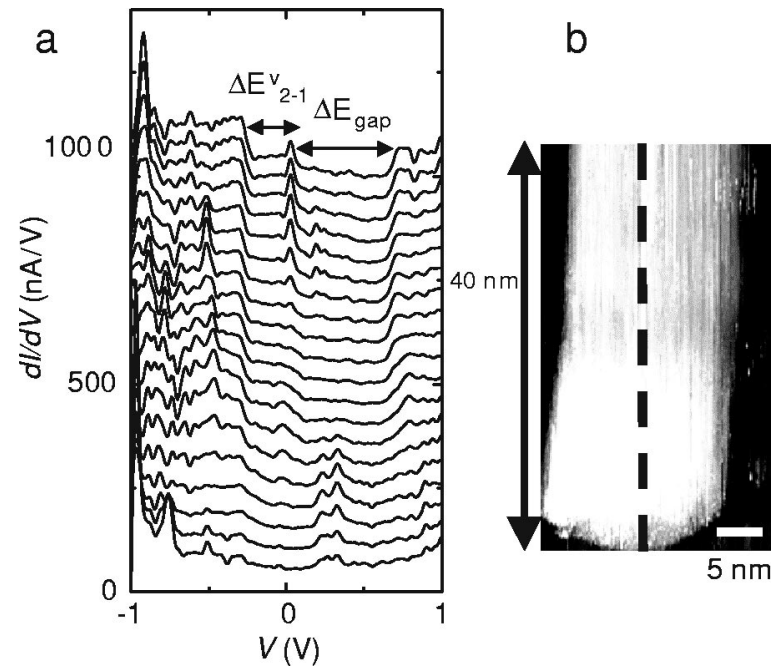

FIG. 5. Electronic structure of the end of a semiconducting nanotube. (a) $d I / d V$ spectra measured at positions $2 \mathrm{~nm}$ apart along the dashed line shown in (b). The energy gap $\Delta E_{g a p}$ and the subband interval $\Delta E_{2-1}^{v}$ are indicated in the top curve. From top to bottom, the band structure gradually fades out and conductance peaks arise at the end. (b) Topographic image of the nanotube end taken at a bias voltage of $0.4 \mathrm{~V}$.

\section{B. Spatially resolved spectroscopy}

\section{Nanotube ends}

By measuring $d I / d V$ as a function of position in the length direction of a nanotube, the evolution of the DOS near special sites such as nanotube ends can be observed. The straight-dotted line drawn in Fig. 1(d) crossing the end of a nanotube, is an example of such a measurement. Figure 5(a) displays a series of curves, measured on a semiconducting nanotube, each about $2 \mathrm{~nm}$ apart along the dashed line indicated in the topographic image of Fig. 5(b). The curves are offset vertically to show the gradual change of the tunneling DOS along the tube near the end. Starting form the top, the band structure can be well observed and is reproducible for adjacent curves. The energy gap $\Delta E_{g a p}$ and the first subband separation $\Delta E_{2-1}^{v}$ are indicated for the top curves. Near the tube end, the subband edges fade out and two new peaks appear within the energy gap at about $0.3 \mathrm{~V}$. These peaks also fade out again in the last two curves where the STM moves off the tube end and onto the gold substrate. In the topographic image of Fig. 5(b), which is obtained at a bias voltage of $0.4 \mathrm{~V}$, the nanotube appears brighter near the end. This corresponds to the observed higher local density-ofstates within the energy gap in the last $\sim 10 \mathrm{~nm}$.

A very illustrative way of presenting the spatially resolved data is to plot $d I / d V$ in grayscale as a function of both the voltage $V$ and the position $x$ along the nanotube. Such a grayscale graph is shown in Fig. 6(a) for a spectroscopy measurement on another semiconducting nanotube. $d I / d V$ curves are obtained about $0.15 \mathrm{~nm}$ apart along a line of $30 \mathrm{~nm}$. At the top, where $x=0$, the STM tip is far away from the end. After about $25 \mathrm{~nm}$, the tip moves off the tube and onto the gold. A dark color in this graph corresponds to a high-tunneling conductance. Figure 6(b) shows a few $d I / d V$ curves taken from horizontal cross sections in Fig. $6(\mathrm{a})$, at the positions 1,2 , and 3 that are indicated in the
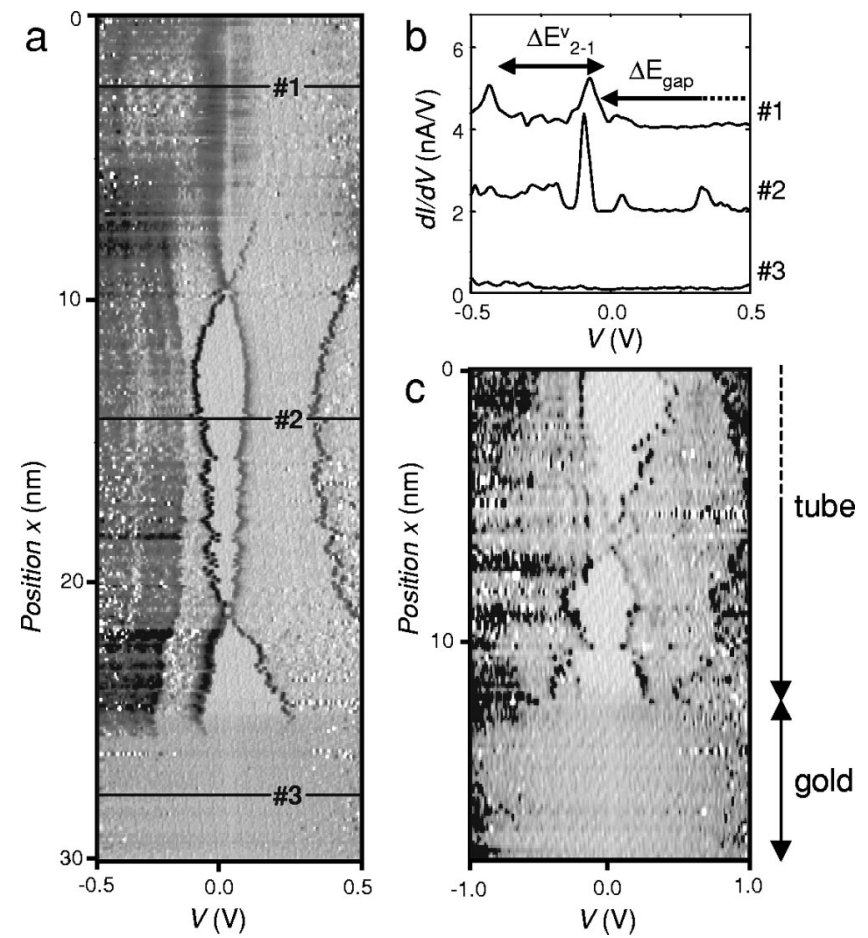

FIG. 6. Tunneling conductance measured as a function of both the bias voltage and the position along ends of semiconducting nanotubes. (a) $d I / d V$ in grayscale plotted against the voltage $V$ and position $x$ along a nanotube length axis. Dark colors correspond to large tunneling conductance. In the bulk of the nanotube $(x<8$ $\mathrm{nm}$ ), the STM tip is far away from the end and the local DOS is similar to the semiconducting band structure. For $x>8 \mathrm{~nm}$, sharp peaks arise in the $d I / d V$ that fluctuate in energy as a function of position. At the last few nm $(x>26 \mathrm{~nm})$, the STM tip is off the tube. (b) $d I / d V$ curves that are taken from horizontal cross sections in (a) indicated with the numbers 1,2 , and 3 . The curves are offset by $2 \mathrm{nA} / \mathrm{V}$ for clarity. (c) $d I / d V$ in grayscale versus $V$ and $x$ for a different semiconducting nanotube end. The tip is off the tube at the last few nm, where $x>13 \mathrm{~nm}$.

grayscale graph. The $d I / d V$ spectrum measured far away from the tube end, curve 1 , indicates that this nanotube is semiconducting. The energy gap is not completely visible due to the finite $\pm 0.5 \mathrm{~V}$ voltage window, but the strong rise in $d I / d V$ near zero bias corresponds to the onset of the first valence subband observed for all semiconducting curves. The zero-bias anomaly can be clearly observed in the grayscale graph, as the light-gray vertical stripe at $0 \mathrm{~V}$ for 0 $<x<8 \mathrm{~nm}$. The most striking feature is the fading out of the band structure at $x \approx 8 \mathrm{~nm}$ and the appearance of three sharp peaks that show an oscillating behavior. These three $d I / d V$ peaks can, for example, also be seen in curve 2. In the bottom few $\mathrm{nm}$, for $x>25 \mathrm{~nm}$, the STM tip actually measures $d I / d V$ on the gold substrate. The tunneling conductance here is indeed featureless (curve 3). Measurements on the same nanotube, taken several minutes later, showed that the oscillating pattern of $d I / d V$ peaks is highly reproducible. Between measurements, voltage pulses were applied to the STM tip, away from the nanotube, to change the shape of the tip apex. Since this did not change the observed behavior in the nanotube, we can exclude artifacts due to the microscopic shape of the tip. The remarkable behavior of oscillating peaks has also been observed on other semiconducting 
nanotubes. An example is shown in Fig. 6(c) for a different semiconducting nanotube. Again, sharp peaks are observed that fluctuate along the tube.

We have no good understanding of all the observed features in $d I / d V$ at the nanotube ends. It would be useful to know the geometry of the ends, for example, whether they are closed or open. The single-walled nanotubes are assumed to be closed by caps during the growth, but it is difficult to confirm this by imaging because of the bluntness of the STM tip. In fact, STM spectroscopy can in principle be used to identify the nanotube ends ${ }^{9,36}$ as has been done by Carrol et al. ${ }^{9}$ and Kim et al. ${ }^{14}$ They measured sharp resonances in the tunneling conductance at the last few $\mathrm{nm}$ at the end of nanotubes and identified these as localized states corresponding to a specific arrangement of pentagons at the end. In our case, sharp peaks also appear but they are found to oscillate in energy position over a large distance, $10-20 \mathrm{~nm}$, which cannot be explained by the presence of localized defects alone. Possibly, the gradually changing capacitance between the STM tip and nanotube when the tip scans over the end has to be taken into account. Simulations ${ }^{37}$ show that the capacitance between the tube and tip changes at the end over a length scale corresponding to the tip size. Our STM tips are typically $10-20 \mathrm{~nm}$, which is also the scale over which the fluctuation patterns are observed. However, when the tip is dispaced a few $\mathrm{nm}$, the change in capacitance should be of the order of a few $10^{-3} \mathrm{aF}$, which cannot explain the large energy fluctuations of about $0.1 \mathrm{eV}$ within that distance.

\section{Nanotube kink}

As discussed in Sec. II, nanotubes are often continuously bent due to elastic deformations. However, sharp bends or kinks may also appear due to junctions between two nanotubes with different $(n, m)$ indices. Such junctions can be possible when pentagon-heptagon defect pairs are present in the hexagonal carbon network. ${ }^{38-40}$ In principle, nanotubes with a different electronic character can be connected to each other by such kinks, which is of interest for nanoscale electronics. Nanotube kinks have indeed been observed by TEM (Refs. 41 and 42) and atomic force microscope (AFM) (Ref. 43) imaging. A recent report of the electronic conductance of nanotube kinks provided evidence for the existence of semiconductor-metal and metal-metal heterojunctions. ${ }^{43}$ Kinklike sharp bends are occasionally observed in STM topographic images [for example Fig. 1(c)]. Figure 6(a) shows a zoomed image of such a sharply bent nanotube. At various positions, indicated by the numbers $1-5$, STS measurements were done. The tunnel spectra above the kink are metallic [curves 1 and 2 in Fig. 7(b)] whereas below the kink they are semiconducting (curves 3-5). This result strongly suggests that the kink is a heterojunction between a semiconducting and metallic nanotube.

\section{SUMMARY}

In this paper we surveyed various measurements of the tunneling DOS in single-walled carbon nanotubes on
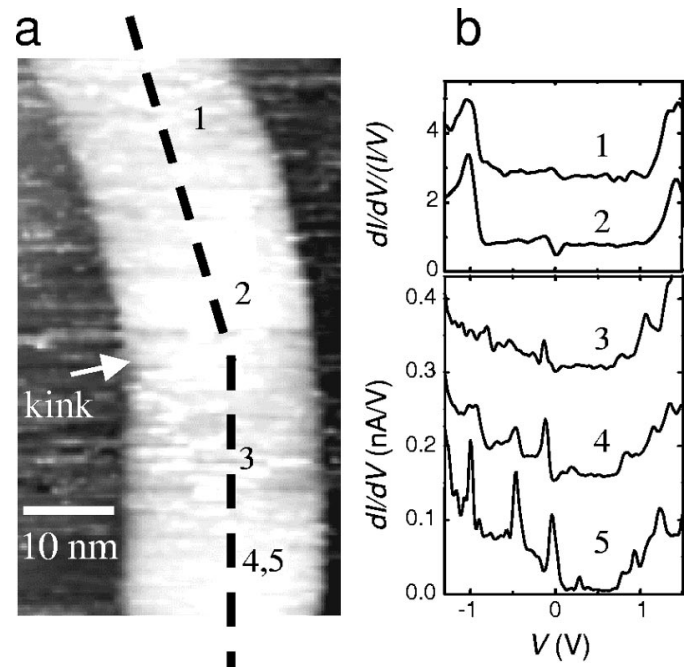

FIG. 7. Topography and spectroscopy measurements on a nanotube kink that appears to be a semiconductor-metal junction. (a) Topographic image of the nanotube kink. The bend angle is $17^{\circ}$. Numbers $1-5$ indicate positions where spectroscopy measurements were done. (b) $d I / d V$ and normalized $d I / d V$ curves measured at positions $1-5$. These curves indicate metallic behavior of the top part $(1,2)$ and semiconducting behavior of the bottom part $(3-5)$. Curve 1 is offset by 2 , curves 3 and 4 by $0.15 \mathrm{nA} / \mathrm{V}$.

$\mathrm{Au}(111)$. From $d I / d V$ tunnel spectra, all nanotubes with various chiralities could be classified as either metallic or semiconducting, with energy subband separations that compare well to the theoretical predictions. Discrete energy levels could be measured in the local DOS at low energies for a short metallic nanotube. The measured DOS curves are positioned asymmetrically around zero bias, indicating a shift of the Fermi level towards lower energies. This can be attributed to the difference in work function between the nanotubes and the gold substrate.

Spatially resolved measurements are used to observe changes in the local DOS at interesting topological sites such as nanotube ends and kinks. Measurements on semiconducting tube ends show that sharp conductance peaks appear within the energy gap that fluctuate in energy with position along the nanotube axis in a reproducible pattern. We do not yet have an explanation for all the experimental facts. We obtained also spectroscopy data on a nanotube kink that appears to be a molecular semiconducting-metal heterojunction. These measurements show that spatially resolved STM spectroscopy can be a useful technique to study the electronic structure of carbon nanotubes and may be exploited further to characterize nanotube devices such as kink heterojunctions.

\section{ACKNOWLEDGMENTS}

We thank Richard Smalley and co-workers for supplying the nanotube material and Philippe Lambin for discussions and providing the theoretical DOS curves. This work was supported by the Dutch Foundation for Fundamental Research of Matter (FOM). 
*Present address: Department of Physics, University of Basel, Klingelbergstrasse 82, CH-4056 Basel, Switzerland

${ }^{1}$ C. Dekker, Phys. Today 52(5), 22 (1999).

${ }^{2}$ J.W. Mintmire, B.I. Dunlap, and C.T. White, Phys. Rev. Lett. 68, 631 (1992).

${ }^{3}$ N. Hamada, S. Sawada, and A. Oshiyama, Phys. Rev. Lett. 68, 1579 (1992).

${ }^{4}$ R. Saito, M. Fujita, G. Dresselhaus, and M.S. Dresselhaus, Appl. Phys. Lett. 60, 2204 (1992).

${ }^{5}$ Z. Zhang and C. Lieber, Appl. Phys. Lett. 62, 2792 (1993).

${ }^{6}$ M. Ge and K. Sattler, Appl. Phys. Lett. 65, 2284 (1994).

${ }^{7}$ C. Olkand and J. Heremans, J. Mater. Res. 9, 259 (1994).

${ }^{8}$ W. Rivera, J.M. Perez, R.S. Ruoff, D.C. Lorents, R. Malhorta, S. Lim, Y.G. Rho, E.G. Jacobs, and R.F. Pinizzotta, J. Vac. Sci. Technol. B 13, 327 (1995).

${ }^{9}$ D.J. Carrol, P. Redlich, P.M. Ajayan, J. C. Charlier, X. Blase, A. De Vita, and R. Car, Phys. Rev. Lett. 78, 2811 (1997).

${ }^{10}$ J. Wildoer, L. Venema, A. Rinzler, R. Smalley, and C. Dekker, Nature (London) 39, 59 (1998).

${ }^{11}$ T. Odom, J.-L. Huang, P. Kim, and C. Lieber, Nature (London) 391, 62 (1998).

${ }^{12}$ W. Clauss, D.J. Bergeron, and A.T. Johnson, Phys. Rev. B 58, R4266 (1998).

${ }^{13}$ A. Hassanien, M. Tokumoto, Y. Kumazawa, H. Kataura, Y. Maniwa, S. Suzuki, and Y. Achiba, Appl. Phys. Lett. 73, 3839 (1998).

${ }^{14}$ P. Kim T. Odom, J.-L. Huang, and C.M. Lieber, Phys. Rev. Lett. 82, 1225 (1999).

${ }^{15}$ C.T. White, and J.W. Mintmire, Nature (London) 394, 29 (1998).

${ }^{16}$ J.-C. Charlier and Ph. Lambin, Phys. Rev. B 57, R15 037 (1998).

${ }^{17}$ A. Thess, R. Lee, P. Nikolaev, H. Dai, P. Petit, J. Robert, C. Xu, Y.H. Lee, S.G. Kim, A.G. Rinzler, D.T. Colbert, G.E. Scuseria, D. Tománek, J.E. Fischer, and R.E. Smalley, Science 273, 483 (1996).

${ }^{18}$ T. Hertel, R.E. Walkup, and Ph. Avouris, Phys. Rev. B 58, 13870 (1998).

${ }^{19}$ A. Rubio, Appl. Phys. A: Mater. Sci. Process. 68, 275 (1999).

${ }^{20}$ V. Meunier and Ph. Lambin, Phys. Rev. Lett. 81, 5888 (1998).

${ }^{21}$ L.C. Venema, V. Meunier, Ph. Lambin, and C. Dekker, Phys. Rev. B 61, 2991 (2000).

${ }^{22} \mathrm{G}$. Dresselhaus et al. (unpublished).

${ }^{23}$ J.A. Stroscio and R.M. Feenstra, in Scanning Tunneling Microscopy, Vol 27 of Methods in Experimental Physics, edited by J.A. Stroscio and W.J. Kaiser (Academic Press, San Diego, 1993),
Chap. 4 and references therein.

${ }^{24}$ P. Chen, X. Wu, S.J. Lin, W. Ji, and K.L. Tan, Phys. Rev. Lett. 82, 2548 (1999).

${ }^{25}$ H. Ago, T. Kugler, F. Cacialli, W.R. Salaneck, M.S.P. Shaffer, A.H. Windle, and R.H. Friend J. Phys. Chem. B 103, 8116 (1999).

${ }^{26}$ Y. Xue and S. Datta, Phys. Rev. Lett. 83, 4844 (1999); cond-mat/9908073 (unpublished).

${ }^{27}$ B.L. Altshuler and A.G. Aronov, Solid State Commun. 30, 115 (1979).

${ }^{28}$ B.L. Altshuler, A.G. Aronov, and P.A. Lee, Phys. Rev. Lett. 44, 1288 (1980).

${ }^{29}$ G.-L. Ingold and Yu.V. Nazarov, in Single Charge Tunneling, NATO ASI Series B, Vol 294, edited by H. Grabert and M.H. Devoret (Plenum Press, New York, 1991), Chap. 2.

${ }^{30}$ R. Egger and A. Gogolin, Phys. Rev. Lett. 397, 5082 (1997).

${ }^{31}$ C. Kane, L. Balents, and M.P.A. Fisher, Phys. Rev. Lett. 79, 5086 (1997).

${ }^{32}$ S.J. Tans, M.H. Devoret, H. Dai, A. Thess, R.E. Smalley, L.J. Geerligs, and C. Dekker, Nature (London) 386, 474 (1997).

${ }^{33}$ M. Bockrath, D.H. Cobden, P.L. McEuen, N.G. Chopra, A. Zettl, A. Thess, and R.E. Smalley, Science 275, 1922 (1997).

${ }^{34}$ L.C. Venema, J.W.G. Wildoer, J.W. Janssen, S.J. Tans, H.L.J. Temminck Tuinstra, L.P. Kouwenhoven, and C. Dekker, Science 283, 52 (1999).

${ }^{35}$ L.C. Venema, J.W.G. Wildöer, H.L.J. Temminck Tuinstra, and C. Dekker, Appl. Phys. Lett. 71, 2629 (1997).

${ }^{36}$ A. DaVita, J.-Ch. Charlier, X. Blase, and R. Car, Appl. Phys. A: Mater. Sci. Process. 68, 283 (1999).

${ }^{37}$ The program used for the capacitance simulations is FastCap, developed by the Research Laboratory of Electronics at MIT, 1992.

${ }^{38} \mathrm{Ph}$. Lambin, A. Fonseca, J.P. Vigneron, J.B. Nagy, and A.A. Lucas, Chem. Phys. Lett. 245, 85 (1995).

${ }^{39}$ R. Saito, G. Dresselhaus, and M.S. Dresselhaus, Phys. Rev. B 53, 2044 (1996).

${ }^{40}$ L. Chico, V.H. Crespi, L.X. Benedict, S.G. Louie, and M.L. Cohen, Phys. Rev. Lett. 76, 971 (1996).

${ }^{41}$ S. Iijima, T. Ichihashi, and Y. Ando, Nature (London) 356, 776 (1992).

${ }^{42}$ J. Han, M.P. Anantram, R.L. Jaffe, J. Kong, and H. Dai, Phys. Rev. B 57, 14983 (1998).

${ }^{43}$ Z. Yao, H.W.Ch. Postma, L. Balents, and C. Dekker, Nature (London) 402, 273 (1999). 